\title{
Transcription conventions for the Greek oral data and its translation
}

\author{
/ self-correction \\ // interruption \\ () incomprehensible parts of utterances \\ [] overlapping talk \\ $\mathrm{xxx} \quad$ stressed parts of utterances \\ $\overline{x x::} \mathrm{x}:$ prolongation of a sound \\ $\{x x x\}$ comments and contextual information added by the author \\ falling intonation \\ ongoing intonation \\ rising intonation in the Greek text \\ rising intonation in the English translation
}


